\title{
AN EXTENSION OF THE DOMAIN OF GAMMA FUNCTIONS OF COMPLEX VARIABLES USING ANALYTIC CONTINUATION
}

\author{
${ }^{1}$ Egahi, M., ${ }^{2}$ Agbata, B.C., ${ }^{3}$ Shoir, M.M., \& ${ }^{4}$ Ode, O.J \\ ${ }^{2}$ Department of Mathematics, University of Nigeria, Nsukka, Nigeria \\ DOI: $10.31364 / \mathrm{SCIRJ} / \mathrm{v} 8.15 .2020 . P 0520775$ \\ http://dx.doi.org/10.31364/SCIRJ/v8.i5.2020.P0520775
}

${ }^{1,3,4}$ Department of Mathematics/Computer Science, Benue State University, Makurdi, Nigeria

\begin{abstract}
This article presents an extension of the domain of gamma functions using analytic continuation. We used the process of analytic continuation to extend the gamma function beyond the original domain, the half plane $\operatorname{Re}(z)>0$.We obtained new interesting results involving analytic continuation and the gamma functions with illustrative examples.
\end{abstract}

Keywords: Analytic continuation, gamma function, complex plane, domain, Half plane

\section{INTRODUCTION}

Let $\mu=\{z \in \mathbb{C}:|z|<1\}$ denotes the interior of the open unit disk in the complex plane $\mathbb{C}$ and suppose $f(z)$ is analytic function in $\mu$, the function $f(z)$ exhibits a remarkable phenomenon of action at interval of points. Which implies that the behaviour of analytic function over an interval irrespective of its magnitude, is sufficient to evaluate completely its behaviour at every other points. Furthermore, if the function $f(z)$ satisfies a certain functional relationship in one part of its domain, then this relationship is adequate to determine its behaviour at larger domain. The above process is referred to as analytic continuation and this process can be used to extend the domain of gamma function of complex variables. If a function $f(z)$ is analytic in a larger domain than the domain $\mathrm{D}$ of an analytic function $h(z)$, and still its behaviours agree with those of $h(z)$ within the domain $\mathrm{D}$, we say that $f(z)$ is an analytic continuation of $h(z)[1]$. That is if we have a function which is analytic in a domain $\mathrm{D}$, we can sometimes extend the function to be analytic in a bigger domain.

Definition 1.A function $f(z)$ of the complex variable is said to be analytic at a point $h_{0}$ if its derivatives exists not only at the point $h_{0}$ but at every point within the some neighbourhood of $h_{0}$ [2]

Definition 2. A function $f_{1}(z)$ is analytic in a domain $D_{1}$ and the function $f_{2}(z)$ is analytic in a domain $D_{2}$ then $f_{2}(z)$ is said to be the direct analytic continuation of $f_{1}(z)$ onto $D_{2}$ if $D_{1} \cap D_{2}$ is a non empty set and $f_{1}(z)=f_{2}(z)$ for all $z \in D_{1} \cap D_{2}$

Definition 3.Suppose $f(z)$ is analytic in a domain $D_{1}$ and also that $D_{1}$ is contained in a domain $D_{2}$, we say that $f(z)$ can be analytically continued from $D_{1}$ to $D_{2}$ if there exist a function $g(z)$ such that

i. $\quad g(z)$ is analytic in $D_{2}$

ii. $\quad g(z)=f(z)$ for every $z \in D_{1}[4]$

\section{MAIN RESULTS I}

\section{THEOREM 1}

Suppose $f(z)$ is analytic in a domain $D$ and $f(z)=0$ for all points along an arc AB inside $D$ then $f(z)=0$ for all $z \in D$

Proof

Let $z_{0}$ be any point on any line $\mathrm{AB}$, then a circle at $K_{0}$ exists with a centre at $z_{0}$ such that $K_{0} \subset D$ and for any $\in K_{0}, f(z)$ has the Taylor series expansion 


$$
f(z)=f\left(z_{0}\right)+f^{\prime}\left(z_{0}\right)\left(z-z_{0}\right)+\frac{1}{2} f^{\prime \prime}\left(z_{0}\right)\left(z-z_{0}\right)^{2}+\cdots
$$

But

$$
f\left(z_{0}\right)=f^{\prime}\left(z_{0}\right)=f^{\prime \prime}\left(z_{0}\right)=\cdots=0
$$

Therefore for every $\in K_{0}, f(z)=0$

Choosing another arc in $K_{0}$ and similar procedure shows that $f(z)=0$ for all $z \in D$

\section{THEOREM 2}

Suppose $f_{1}(z)$ and $f_{2}(z)$ are analytic in domains $D_{1}, D_{2}$ respectively, then the analytic continuation of $f_{1}(z)$ to $D_{2}$ is unique.

\section{Proof}

Suppose $f_{2}(z)$ and $f_{2}{ }^{\prime}(z)$ are analytic continuations of $f_{1}(z)$ to $D_{2}$ then

$$
f_{1}(z)=f_{2}(z)=f_{2}^{\prime}(z)
$$

for all $z \in D_{1} \cap D_{2}$

Or

$$
\left(f_{2}(z)-f_{2}{ }^{\prime}(z)\right)=0
$$

for all $z \in D_{1} \cap D_{2}$

We now choose any arc $\mathrm{AB}$ in $D_{1} \cap D_{2}$, then for all $z \in \mathrm{AB}$

$\left(f_{2}(z)-f_{2}{ }^{\prime}(z)\right)=0$ for all $z \in \mathrm{AB}$ and from theorem $1\left(f_{2}(z)-f_{2}{ }^{\prime}(z)\right)=0$ for all $z \in D_{2}$

Or $f_{2}(z)=f_{2}{ }^{\prime}(z)$ for all $z \in D_{2}$. Hence analytic continuation of $f_{1}(z)$ to $D_{2}$ is unique.

\section{Example 1}

Given the identity, $\sin ^{2} x+\cos ^{2} x=1$, the process of analytic continuation shows that it holds for all complex values.

Let $D$ be a domain in the $z$ plane containing a part of $x$ axis. Since $\sin z$ and $\cos z$ are analytic in $D$, the function

$$
f(z)=\sin ^{2} z+\cos ^{2} z-1
$$

is also analytic in $D$.Furthermore, $f(z)=0$ along the $x$ axis and by theorem $1, f(z)=0$ everywhere in $D$ or

for all $z \in \mathrm{D}$

$$
\sin ^{2} z+\cos ^{2} z=1
$$

Since (6) holds for an arbitrary domain, it must be true for the entire complex space .

\section{Example 2}

The integral

$$
f_{1}(z)=\int_{0}^{\infty}(1+t) e^{-z t} d t
$$

is convergent for $\operatorname{Re}(z)>0$.We can determine the analytic continuation of $f_{1}(z)$ into the half plane for $\operatorname{Re}(z)<0$. Applying integration by part to (7) we have 


$$
\begin{gathered}
f_{1}(z)=\left[\frac{e^{-z t}}{-z}\right]_{t=0}^{\infty}+\int_{0}^{\infty} t\left(\frac{e^{-z t}}{-z}\right) d t=\left[\frac{e^{-z t}}{-z}-\frac{t e^{-z t}}{z}-\frac{e^{-z t}}{z^{2}}\right]_{t=0}^{\infty} \\
=\frac{1}{z}+\frac{1}{z^{2}}=\frac{1+z}{z^{2}}, \quad \operatorname{Re}(z)<0 .
\end{gathered}
$$

The function $f_{2}(z)=\frac{1+z}{z^{2}}, \quad z \neq 0$ is analytic in the whole complex plane except the origin. Thus $f_{2}(z)$ is the analytic continuation of $f_{1}(z)$ into the left half plane $\operatorname{Re}(z)<0$

\section{THEOREM 3}

Let $f_{1}(z)$ be analytic in some domain $D_{1}$ which includes a segment of the $x$-axis, and which assumes real values on this segment, then the analytic continuation of $f_{1}(z)$ into a domain $D_{2}$ which is the mirror image of $D_{1}$ with respect to the $x$ - axis, is given by

$$
f_{2}(z)=\overline{f_{1}(\bar{z})}
$$

\section{Proof}

Let $\mathrm{AB}$ be a segment on $x$ - axis then

$$
f_{1}(z)=f_{1}(x)=\overline{f_{1}(x)}=\overline{f_{1}(\bar{z})}
$$

We therefore show that $f_{2}(z)=\overline{f_{1}(\bar{z})}$ is analytic in $D_{2}$. Let

$$
f_{1}(z)=u(x, y)+i v(x, y)
$$

The function $f_{1}(z)$ is analytic in $D_{1}$. Hence, by the Cauchy -Riemann equations

$$
\frac{\partial u}{\partial x}=\frac{\partial v}{\partial y}, \quad \frac{\partial v}{\partial x}=-\frac{\partial u}{\partial y}
$$

The partial derivatives above are continuous, we have

and

$$
f_{1}(\bar{z})=f_{1}(x-i y)=u(x,-y)+i v(x,-y)
$$

From equation 12 we obtain

$$
\overline{f_{1}(\bar{z})}=u(x,-y)-i v(x,-y)=f_{2}(z)
$$

$\frac{\partial u}{\partial x}=\frac{\partial(-v)}{\partial(-y)}=\frac{\partial v}{\partial y}$ and $\frac{\partial v}{\partial x}=\frac{\partial(u)}{\partial(-y)}=-\frac{\partial u}{\partial y}$

Therefore $f_{2}(z)$ is analytic. Since on $\mathrm{AB} f_{1}(z)=f_{2}(z)$ and $f_{2}(z)$ is analytic in $D_{2}, f_{2}(z)$ is the analytic continuation of $f_{1}(z)$ into $D_{2}$

\section{THEOREM 4}

Let $\left(z_{n}\right)$ be a sequence of points in $D$ converging to a point $h \neq z_{k}$, and let $f_{1}(z)$ and $f_{2}(z)$ be two functions analytic in $D$. If $f_{1}\left(z_{k}\right)=f_{2}\left(z_{k}\right), k=1,2, \ldots$. Then $f_{1}(z)=f_{2}(z)$ for $z \in \mathrm{D}$

\section{Proof}

Let

$$
f(z)=f_{1}(z)-f_{2}(z)
$$

The function $f(z)$ is analytic at $h$, its Taylor series expansion around $z=h$ is

$$
f(z)=h_{0}+h_{1}\left(z-h_{0}\right)+h_{2}\left(z-h_{0}\right)^{2}+\cdots
$$

Which converges for $|z-h|<r$, we have

$f\left(z_{k}\right)=f_{1}\left(z_{k}\right)-f_{2}\left(z_{k}\right)=0$ for $k=1,2, \ldots$ 
Since $f(z)$ is continuous at $h$ and $\lim _{k \rightarrow \infty} z_{k}=h$ we conclude that $h_{0}=f(h)=0$

We shall show that all other coefficients in the Taylor series are equal to zero. Indeed assume that

$h_{1}=h_{2}=\cdots=h_{\tau-1}=0, h_{\tau} \neq 0$

Then

$$
f(z)=h_{\tau}(z-h)^{\tau}+h_{\tau+1}(z-h)^{\tau+1}+\cdots=F(z)(z-h)^{\tau}
$$

But $f\left(z_{k}\right)=0$ Therefore $F\left(z_{k}\right)=0$

The function $F\left(z_{k}\right)$ is continuous at $h$. Hence, $h_{\tau}=F(h)=0$

All coefficients in the Taylor series vanish, therefore $f(z)=0$ and $f_{1}(z)=f_{2}(z)$ for $z \in \mathrm{D}$

\section{MAIN RESULT II}

\section{Analytic Continuation and the Gamma Function}

The gamma function has its roots in attempt to extend a function beyond certain domain by analytic continuation. It has an impressive number of different representations including limit, series, and integral forms, each offering its own unique advantage in many striking applications. Apart from its dominant role in pure mathematics, the gamma function also plays vital role in applied mathematics like fluid dynamics, mathematical physics, statistics and astrophysics.

The complex gamma function is given by the integral

$$
\Gamma(\mathrm{z})=\int_{0}^{\infty} t^{z-1} e^{-t} d t
$$

which converges absolutely for $\operatorname{Re}(z)>0$

\section{Lemma 1}

The $\Gamma(\mathrm{z})$ is analytic function of $z$ in the half plane $\operatorname{Re}(z)>0$

\section{Proof}

We first show that $\lim _{z_{0} \rightarrow 0} \frac{\Gamma\left(\mathrm{z}+z_{0}\right)-\Gamma(\mathrm{z})}{z_{0}}$ exist. Indeed

$$
\begin{aligned}
\frac{\Gamma\left(\mathrm{z}+z_{0}\right)-\Gamma(\mathrm{z})}{z_{0}} & =\frac{1}{z_{0}} \int_{0}^{\infty} t^{z+z_{0}-1} e^{-t} d t-\frac{1}{z_{0}} \int_{0}^{\infty} t^{z-1} e^{-t} d t \\
& =\int_{0}^{\infty} t^{z-1} e^{-t}\left[\frac{t^{z_{0}}-1}{z_{0}}\right] d t
\end{aligned}
$$

The limit is equal to

$$
\begin{gathered}
\lim _{z_{0} \rightarrow 0} \frac{\Gamma\left(\mathrm{z}+z_{0}\right)-\Gamma(\mathrm{z})}{z_{0}}=\lim _{z_{0} \rightarrow 0} \int_{0}^{\infty} t^{z-1} e^{-t}\left[\frac{t^{z_{0}}-1}{z_{0}}\right] d t \\
=\int_{0}^{\infty} t^{z-1} e^{-t} \ln t d t
\end{gathered}
$$

The limit was evaluated using L'Hospital's rule: 


$$
\lim _{z_{0} \rightarrow 0}=\frac{t^{z_{0}}-1}{z_{0}}=\left[\frac{d}{d z_{0}}\left(\frac{t^{z_{0}}-1}{z_{0}}\right)\right]_{z_{0}=0}=\ln t
$$

Hence, $\Gamma(\mathrm{z})$ is an analytic function in the half- plane $\operatorname{Rez}>0$

\section{Preposition 1}

i. $\quad$ The gamma function $\Gamma(\mathrm{z})$ satisfies $\Gamma(\mathrm{z}+1)=\mathrm{z} \Gamma(\mathrm{z})$

ii. The functional equation (i) extends analytic continuation of $\Gamma(\mathrm{z})$ beyond the original domain $\operatorname{Re}(z)>0$

\section{Proof}

(i) This is known as the recursion formula. The gamma function $\Gamma(\mathrm{z})$ is defined for all $z$ such that for $\operatorname{Re}(z)>0$ $\Gamma(\mathrm{z})=\int_{0}^{\infty} t^{z-1} e^{-t} d t$. Applying integration by part to $\Gamma(\mathrm{z}+1)$, we have

$$
\begin{gathered}
\Gamma(\mathrm{z}+1)=\int_{0}^{\infty} t^{z} e^{-t} d t=\lim _{q \rightarrow \infty} \int_{0}^{q} t^{z} e^{-t} d t \\
=\lim _{q \rightarrow \infty}\left[\left.t^{z}\left(-e^{-t}\right)\right|_{t=0} ^{q}-\int_{0}^{q} z t^{z-1}\left(-e^{-t}\right) d t\right] \\
=z \int_{0}^{\infty} t^{z} e^{-t} d t=\mathrm{z} \Gamma(\mathrm{z})
\end{gathered}
$$

$\operatorname{Re}(z)>0$ where $\lim _{q \rightarrow \infty} \frac{q^{z}}{e^{q}}=0$

(ii) Since (i) holds for $\operatorname{Re}(z)>0$, It must remain valid for all analytic continuation of the function appearing in (i). The function $\Gamma(\mathrm{z}+1)$ can be continued analytically to all point $z$ such that $\operatorname{Re}(z+1)>0$ that is $\operatorname{Re}(z)>-1$. The same argument is true for the function $z \Gamma(\mathrm{z})$. (i) holds for the half plane $\operatorname{Re}(z)>-1$ the value of $\Gamma(\mathrm{z})$ for the strip $-1<\operatorname{Re}(z) \leq 0$ can be obtained from

$$
\Gamma(\mathrm{z})=\frac{\Gamma(\mathrm{z}+1)}{\mathrm{z}}
$$

Initially $\Gamma(\mathrm{z})$ was defined for the domain $\operatorname{Re}(z)>0$.Equation (17) extends the domain of definition to $D_{1},-1<\operatorname{Re}(z) \leq 0$. From (17) we conclude that $\Gamma(\mathrm{z})$ is analytic for all points of the strip except at $z=0$ where it has a simple pole.

In the same way we can add the additional stripes to the domain of definition to obtain $\operatorname{Re}(z)>-n$, where $n$ is an arbitrary positive integer. The function $\Gamma(\mathrm{z})$ is analytic for all points of $\operatorname{Re}(z)>-n$ except for the simple poles located at $z=$ $0,-1,-2,-3, \ldots,(n-1), \ldots$

\section{Preposition 2}

The gamma functions $\Gamma(\mathrm{z})$ satisfies the functional equations

$$
\text { (i) } \quad \Gamma(\mathrm{z})=\frac{\Gamma(\mathrm{z}+\mathrm{m})}{\mathrm{z}(\mathrm{z}+1)(\mathrm{z}+2) . .(\mathrm{z}+\mathrm{m}-1)}
$$

where $m$ is a positive integer

$$
\text { (ii) } \quad \Gamma(\mathrm{z}) \Gamma(1-\mathrm{z})=\frac{\pi}{\sin \mathrm{z}}
$$




\section{Proof}

(i) From preposition 1 part (i) we have that

and

$$
\Gamma(\mathrm{z}+1)=\mathrm{z} \Gamma(\mathrm{z})
$$

Substituting (20) into (21), we have

$$
\Gamma(\mathrm{z}+2)=(\mathrm{z}+1) \Gamma(\mathrm{z}+1)
$$

$$
\Gamma(\mathrm{z}+2)=(\mathrm{z}+1) \mathrm{z} \Gamma(\mathrm{z})
$$

Similarly for $\Gamma(z+3)$

$$
\Gamma(\mathrm{z}+3)=(\mathrm{z}+2) \Gamma(\mathrm{z}+2)=(\mathrm{z}+2)(\mathrm{z}+1) \mathrm{z} \Gamma(\mathrm{z})
$$

In general

$$
\Gamma(\mathrm{z}+\mathrm{m})=(\mathrm{z}+\mathrm{m}-1)(\mathrm{z}+\mathrm{m}-2) \ldots(\mathrm{z}+1) \mathrm{z} \Gamma(\mathrm{z})
$$

(ii) First we shall prove (ii) for $z$ such that $\operatorname{Im} z=0$ and $0<\operatorname{Rez}<1$, and

then by analytic continuation we will extend the result to other values of $z$. But

$$
\begin{aligned}
\Gamma(\mathrm{z}) \Gamma(1-\mathrm{z}) & =\left[2 \int_{0}^{\infty} \mathrm{x}^{2 \mathrm{~m}-1} \mathrm{e}^{-\mathrm{x}^{2}} \mathrm{dx}\right]\left[2 \int_{0}^{\infty} \mathrm{y}^{1-2 \mathrm{~m}} \mathrm{e}^{-\mathrm{y}^{2}} \mathrm{dy}\right] \\
& =4 \int_{0}^{\infty} \int_{0}^{\infty} \mathrm{x}^{2 \mathrm{~m}-1} \mathrm{y}^{1-2 \mathrm{~m}} \mathrm{e}^{-\left(\mathrm{x}^{2}+\mathrm{y}^{2}\right)} \mathrm{dxdy}
\end{aligned}
$$

In polar coordinate $x=r \cos \theta$ and $y=r \sin \theta$ so that

$$
\begin{gathered}
\Gamma(\mathrm{z}) \Gamma(1-\mathrm{z})=4 \int_{\theta}^{\frac{\pi}{2}} \int_{r=0}^{\theta}\left(\tan ^{1-2 m} \theta\right)\left(r e^{-r^{2}}\right) d r d \theta \\
=2 \int_{\theta}^{\frac{\pi}{2}} \tan ^{1-2 m} \theta d \theta=\frac{\pi}{\sin m \pi}
\end{gathered}
$$

We have used the integral below to get (22):

$$
\int_{0}^{\infty} \frac{x^{\alpha-1}}{x+1} d x=\frac{\pi}{\sin \alpha \pi} \text { for } 0<\alpha<1
$$

Thus $\Gamma(\mathrm{z}) \Gamma(1-\mathrm{z})=\frac{\pi}{\sin \alpha \pi}$.

\section{CONCLUSION}

In complex analysis, most functions are defined on a small domain only before extending its behaviour by analytic continuation which is often done by first establishing some functional equation on the small domain and then this equation can now be applied in extending it to other larger domain. The process of analytic continuation has applications in other areas of mathematics like Riemann Zeta function, development of Riemann surfaces, solution of boundary value problems, construction of potential energy curves. 


\section{REFERENCES}

1. Lars Ahlfors(1979). Complex Analysis. 3edn, McGraw-Hill. Pp172,284.

2. P. Dienes (1957). The Taylor series: An introduction to the theory of functions of a complex variable. New York:Dover publications, Inc

3. Jeremy Orloff (2010). Notes on analytic continuation and the gamma function

4. E.B. Saff and A.D. Snider,(2003). Fundamentals of Complex Analysis with Applications to Engineering and Science, $3^{\text {rd }}$ Edition, Prentice Hall.

5. N.N. Lebedev, (1965). Special Functions and their Applications, Prentice Hall, New Jersey 